\title{
ON THE VARIANCES OF OCCUPATION TIMES OF CONDITIONED BROWNIAN MOTION
}

\author{
BIAO ZHANG
}

\begin{abstract}
We extend some bounds on the variance of the lifetime of twodimensional Brownian motion, conditioned to exit a planar domain at a given point, to certain domains in higher dimensions. We also give a short "analytic" proof of some existing results.
\end{abstract}

\section{INTRODUCTION}

This paper studies questions related to the variance of the lifetime of certain $h$-processes. Our estimates are related to a result of B. Davis, stated below (Theorem 1.1), and in fact we give a short, "analytic" proof of this result. $h$-processes are intimately connected with many aspects of partial differential equations and harmonic analysis, and in particular variance estimates have recently been used to study intrinsic ultracontractivity, in [1] and [4].

If $A$ is a Borel subset of $\mathbb{R}^{d}, d \geq 2$, the Lebesgue measure, closure, complement, and Euclidean boundary of $A$ are respectively denoted by $|A|, \bar{A}, A^{c}$, and $\partial A$. Let $D$ be a domain of $\mathbb{R}^{d}$, which has a Green function, let $P_{x}$ and $E_{x}$ be probability and expectation of standard $d$-dimensional Brownian motion started at $x$, and let $P_{x}^{y}$ and $E_{x}^{y}$ denote the probability and expectation of this motion either conditioned to exit $D \backslash\{y\}$ at $y$, if $y \in D$, or conditioned to exit $D$ at the point $y$ in its minimal Martin boundary $\Delta$, if $y \in \Delta$. Formally, these are the $h$-processes with $h$ respectively the Green function of $D$, denoted by $G(\cdot, y)$, or the Martin kernel function of $D$, denoted by $K(\cdot, y)$. These are the basic $h$-processes in the sense that all the other $h$-processes are mixtures of them, see [3] as a reference. We will discuss $h$-processes in more detail later. We use $\tau_{D}$ to designate the first exit time of a process from $D$, and often shorten $\tau_{D}$ to $\tau$. Positive constants $c, C, c_{M}, C_{M}$ may depend on the dimension and are not necessarily the same at each occurrence. The letters $x$ and $y$ are used to designate respectively the starting and exit points of motions.

By a cube $Q$ of $\mathbb{R}^{d}, d \geq 2$, we always mean a closed cube. A Whitney decomposition of $D$, denoted by $W(D)=\left\{Q_{i}\right\}_{i \geq 0}$, is a collection of closed cubes in $D$ with disjoint interiors, with union $D$, satisfying, for all $i$,

$$
1 \leq \frac{d\left(Q_{i}, \partial D\right)}{\ell\left(Q_{i}\right)} \leq 4 \sqrt{2}
$$

Received by the editors October 24, 1994.

1991 Mathematics Subject Classification. Primary 60J65, 60J05.

Key words and phrases. Conditioned Brownian motion, $h$-processes. 
See [10] for a proof that all domains have a Whitney decomposition. Here $\ell\left(Q_{i}\right)$ is the side length of $Q_{i}$ and $d(\cdot, \cdot)$ is the Euclidean distance between points, sets, or points and sets. The Whitney decomposition gives rise to a quasi-hyperbolic distance in $D$ in the following way. Fix $Q, Q^{\prime} \in W(D)$. We say that $Q=Q_{0} \rightarrow$ $Q_{1} \rightarrow \ldots \rightarrow Q_{m}=Q^{\prime}$ is a Whitney chain connecting $Q$ and $Q^{\prime}$ of length $m$ if $Q_{i} \in W(D)$ for all $i$ and if $Q_{i-1} \cap Q_{i} \neq \varnothing, 1 \leq i \leq m$. Define the Whitney distance $\rho_{D}\left(Q, Q^{\prime}\right)$, or briefly $\rho\left(Q, Q^{\prime}\right)$, to be the length of the shortest Whitney chain connecting $Q$ and $Q^{\prime}$. See [8] for a reference. If $Q \in W(D)$, we denote $\int_{0}^{\tau} I\left(Z_{t} \in Q\right) d t$, the total time $Z_{t}$ spends in $Q$, by $T_{Q}$, and let $P_{Q}=P_{x}^{y}\left(\tau_{Q^{c}}<\tau\right)$ stand for the probability $Z_{t}$ ever hits $Q$, where $\tau_{Q^{c}}$ is the first time $Z_{t}$ hits $Q$. By $\operatorname{cov}\left(T_{Q_{i}}, T_{Q_{j}}\right)=\operatorname{cov}_{x}^{y}\left(T_{Q_{i}}, T_{Q_{j}}\right)$ we mean the covariance of $T_{Q_{i}}$ and $T_{Q_{j}}$ with respect to $P_{x}^{y}$. The following result is due to Burgess Davis [3].

Theorem 1.1. If $Q$ and $R$ are Whitney cubes of a Whitney decomposition of a simply connected planar domain $D$, then if $x \in D$ and $y \in \Delta$,

$$
\left|\operatorname{cov}\left(T_{Q}, T_{R}\right)\right| \leq C e^{-c \rho(Q, R)}|Q||R|\left(P_{Q}+P_{R}\right) .
$$

Davis' proof of this theorem has a substantial probabilistic component and is somewhat involved. Here we first prove the following formula for $\operatorname{cov}\left(T_{Q}, T_{R}\right)$ in terms of $K$ and $G$, for $y \in \Delta$. Let $Q$ and $R$ be subdomains of $D$ which have disjoint interiors. Then

$$
\begin{array}{r}
\operatorname{cov}\left(T_{Q}, T_{R}\right)=\int_{Q} \int_{R}\left\{G(q, r)\left[G(x, r) \frac{K(q, y)}{K(x, y)}+G(x, q) \frac{K(r, y)}{K(x, y)}\right]\right. \\
\left.-G(x, r) G(x, q) \frac{K(q, y) K(r, y)}{K(x, y)^{2}}\right\} d q d r \\
x \in D, y \in \Delta .
\end{array}
$$

We use this formula to prove Theorem 1.1 as well as the following theorem, for domains in $\mathbb{R}^{d}, d \geq 2$, of the form

$$
D=D_{f}=\left\{x=\left(x_{1}, \ldots, x_{d}\right): x_{d}>f\left(x_{1}, \ldots, x_{d-1}\right)\right\},
$$

where $f$ is a Lipschitz function on $\mathbb{R}^{d-1}$. We let $M(f)=M$ stand for the Lipschitz constant of $f$.

Theorem 1.2. If $Q$ and $R$ are Whitney cubes of $D=D_{f}$ with disjoint interiors, then

$$
\left|\operatorname{cov}\left(T_{Q}, T_{R}\right)\right| \leq C_{M} e^{-c_{M} \rho(Q, R)}|Q|^{2 / d}|R|^{2 / d}\left(P_{Q}+P_{R}\right) .
$$

Clearly, Theorem 1.1 can be used in some cases to bound the variance of $\sum T_{\Gamma_{i}}=$ $\int_{0}^{\tau} I\left(Z_{s} \in \bigcup \Gamma_{i}\right) d s$, using the formula,

$$
\operatorname{var}\left(\sum T_{\Gamma_{i}}\right)=\sum \operatorname{var} T_{\Gamma_{i}}+\sum_{i \neq j} \operatorname{cov}\left(T_{\Gamma_{i}}, T_{\Gamma_{j}}\right),
$$

where $\Gamma_{i}$ are Whitney cubes with disjoint interiors. See [1], [3], and [4] for examples. Theorem 1.2 can be similarly employed. Our proofs, unchanged, prove the analogous theorems about Brownian motion conditioned to exit a domain minus a point at that point. See [3] for a description of these processes. We then indicate in a brief paragraph how this proof can be modified to prove Theorem 1.1. See [7] for a proof that the Martin boundary of $D_{f}$ is the Euclidean boundary of $D_{f}$. 


\section{Covariance FORMula}

In this section, (1.1) and some lemmas will be proved. The $\sigma$-fields of a process $Z_{t}, t \geq 0$, are denoted by $\mathcal{F}(u)=\sigma\left(Z_{s}, s \leq u\right)$. If $g$ is a positive harmonic function on $\Gamma$, a domain of $\mathbb{R}^{d}, d \geq 2$, then the $h$-process in $\Gamma$ associated with $g$ is determined by the following transition density function:

$$
p_{t}^{g}(x, y)=\frac{g(y)}{g(x)} p_{t}(x, y)
$$

The corresponding probability and expectation are denoted by $P_{x}^{g}$ and $E_{x}^{g}$ respectively. See [6] for more information on $h$-processes. Here we recall that an $h$-process is a strong Markov process with continuous paths up to its lifetime $\tau_{\Gamma}$, and if $\eta$ is a stopping time of this process and $A \in \mathcal{F}(\eta)$, then

$$
P_{x}^{g}\left(A \cap\left\{\eta<\tau_{\Gamma}\right\}\right)=\int_{A \cap\left\{\eta<\tau_{\Gamma}\right\}} \frac{g\left(Z_{\eta}\right)}{g(x)} d P_{x} .
$$

We define, for any real number $a$,

$$
\begin{aligned}
D_{a} & =\left\{\left|x_{i}\right|<2^{a}, \quad 1 \leq i \leq d\right\} \cap D \text { and } \\
L_{a} & =\left\{\left|x_{i}\right|=2^{a}, 1 \leq i \leq d\right\} \cap D .
\end{aligned}
$$

And we always assume that $0 \in \partial D$ which is the center of our "rectangles" $D_{a}$. It is easy to see that all $D_{a}$ are simply connected Lipschitz domains with Lipschitz constants that can be bounded by a number that depends only on $M>0$, the Lipschitz constant of $f$, that will be denoted by $M$ again. Let $\tau_{a}=\inf \left\{t: Z_{t} \in L_{a}\right\}$ be the first time $Z_{t}$ hits $L_{a}$. By $\operatorname{cov}_{x}^{g}\left(T_{Q}, T_{R}\right)$ we mean the covariance of $T_{Q}$ and $T_{R}$ with respect to $P_{x}^{g}$.

Theorem 2.1. Let $g$ be a positive harmonic function on a Greenian domain $\Gamma \subseteq$ $\mathbb{R}^{d}, d \geq 2$, and $Q$ and $R$ be subdomains of $\Gamma$ having disjoint interiors, then

$$
\begin{array}{r}
\operatorname{cov}_{x}^{g}\left(T_{Q}, T_{R}\right)=\int_{Q} \int_{R}\left\{G_{\Gamma}(q, r)\left[G_{\Gamma}(x, r) \frac{g(q)}{g(x)}+G_{\Gamma}(x, q) \frac{g(r)}{g(x)}\right]\right. \\
\left.-G_{\Gamma}(x, r) G_{\Gamma}(x, q) \frac{g(q) g(r)}{g(x)^{2}}\right\} d q d r .
\end{array}
$$

Proof. By (2.1),

$$
\begin{aligned}
E_{x}^{g} T_{Q} & =E_{x}^{g} \int_{0}^{\tau_{\Gamma}} I\left(Z_{t} \in Q\right) d t \\
& =\int_{0}^{\infty} \int_{Q} p_{t}^{g}(x, q) d q d t \\
& =\int_{Q} G_{\Gamma}(x, q) \frac{g(q)}{g(x)} d q .
\end{aligned}
$$

Similarly,

$$
E_{x}^{g} T_{R}=\int_{R} G_{\Gamma}(x, r) \frac{g(r)}{g(x)} d r
$$


Since

$$
\begin{aligned}
E_{x}^{g} T_{Q} T_{R}= & E_{x}^{g} \int_{0}^{\tau_{\Gamma}} \int_{0}^{\tau_{\Gamma}} I\left(Z_{t} \in Q\right) I\left(Z_{s} \in R\right) d t d s \\
= & \int_{0}^{\infty} \int_{0}^{\infty} P_{x}^{g}\left(Z_{t} \in Q, Z_{s} \in R, t<\tau_{\Gamma}, s<\tau_{\Gamma}\right) d s d t \\
= & \iint_{s \leq t}\left[\int_{Q} \int_{R} p_{s}^{g}(x, r) p_{t-s}^{g}(r, q) d q d r\right] d s d t \\
& +\iint_{s>t}\left[\int_{Q} \int_{R} p_{t}^{g}(x, q) p_{s-t}^{g}(q, r) d q d r\right] d s d t \\
= & \int_{Q} \int_{R}\left\{\int_{0}^{\infty}\left[\int_{s}^{\infty} p_{t-s}^{g}(r, q) d t\right] p_{s}^{g}(x, r) d s\right\} d q d r \\
& +\int_{Q} \int_{R}\left\{\int_{0}^{\infty}\left[\int_{t}^{\infty} p_{s-t}^{g}(q, r) d s\right] p_{t}^{g}(x, q) d t\right\} d q d r \\
= & \int_{Q} \int_{R} G_{\Gamma}(r, q) \frac{g(q)}{g(r)} G_{\Gamma}(x, r) \frac{g(r)}{g(x)} d q d r \\
& +\int_{Q} \int_{R} G_{\Gamma}(q, r) \frac{g(r)}{g(q)} G_{\Gamma}(x, q) \frac{g(q)}{g(x)} d q d r \\
= & \int_{Q} \int_{R} G_{\Gamma}(q, r)\left[G_{\Gamma}(x, r) \frac{g(q)}{g(x)}+G_{\Gamma}(x, q) \frac{g(r)}{g(x)}\right] d q d r,
\end{aligned}
$$

the theorem follows easily from

$$
\operatorname{cov}_{x}^{g}\left(T_{Q}, T_{R}\right)=E_{x}^{g} T_{Q} T_{R}-E_{x}^{g} T_{Q} E_{x}^{g} T_{R} .
$$

Taking $g(x)=K(x, y)$ in Theorem 2.1, for $y \in \Delta$, gives (1.1).

We employ the boundary Harnack principle for Lipschitz domains in the proof of the next lemma. See Jerison and Kenig [9] for a statement of this principle. We will use not only this principle but also the following consequence. We let $x_{0}$ be the reference point of our kernel functions such that $d\left(x_{0}, \partial D_{f}\right) \geq \frac{1}{M}$.

Lemma 2.0. Let $u$ and $v$ be positive and harmonic functions in $D_{2}$. Suppose there are positive constants $c$ and $C$ such that

$$
c<\frac{u(r)}{v(r)}<C,
$$

where $r$ is the point of $\partial D_{0}$ directly above $y$. Suppose that $u$ and $v$ have boundary limits 0 at each point of $\partial D_{f} \cap \partial D_{2}$ except $y$. Then there are constants $c_{M}$ and $C_{M}$ which depend only on $c, C$, and $M$, such that

$$
c_{M}<\frac{u(z)}{v(z)}<C_{M}, \quad z \in \partial D_{1} \backslash \partial D_{f} .
$$

Proof. For points $z$ not too close to $\partial D_{f}$, we use the Harnack inequality. The truth of (2.3) for these points, together with the boundary Harnack principle, gives its truth for all $z \in \partial D_{1} \backslash \partial D_{f}$. 
Lemma 2.1. There exists an integer $n_{0}=n_{0}(M)$, such that

(i) for any $x$ and $y$ inside $L_{0}$ and for any positive integer $m$, we have

$$
P_{x}^{y}\left(\tau_{m n_{0}}<\tau\right) \leq \frac{1}{2^{m}}
$$

(ii) for $x$ and $y$ outside $L_{m n_{0}}$, we have

$$
P_{x}^{y}\left(\tau_{0}<\tau\right) \leq \frac{1}{2^{m}}
$$

Proof. Proof of (i):

It is enough to show that there exists an integer $n_{0}=n_{0}(M)$ such that

$$
P_{x}^{y}\left(\tau_{n_{0}}<\tau\right) \leq \frac{1}{2} \text {, for any } x \text { and } y \text { inside } L_{0},
$$

since the general case follows from this by iteration and scaling.

Let $K_{2}$ be the kernel for $D_{2}$. We have by Lemma 2.0, with

$$
u(x)=\frac{K_{2}(x, z)}{K_{2}(r, z)} \text { and } v(x)=\frac{K(x, y)}{K(r, y)},
$$

where $z$ is a fixed point of $L_{2}$, that

$$
\frac{1}{M} \frac{K_{2}(x, z)}{K(x, y)} \leq \frac{K_{2}(r, z)}{K(r, y)} \leq M \frac{K_{2}(x, z)}{K(x, y)}, x \in \partial D_{1} \backslash \partial D_{f} .
$$

Furthermore, if $\omega_{z}$ denotes harmonic measure on $\partial D_{2}$ with respect to $D_{2}$ and if $x \in \partial D_{1} \backslash \partial D_{f}$ and $j \geq 2$, Lemma 2.0 gives

$$
\begin{aligned}
P_{x}^{y}\left(\text { ever hit } L_{j}\right) & =\int_{L_{2}} P_{z}^{y}\left(\text { ever hit } L_{j}\right) \frac{K(z, y)}{K(x, y)} d \omega_{x}(z) \\
& =\int_{L_{2}} P_{z}^{y}\left(\text { ever hit } L_{j}\right) K(z, y) \frac{K_{2}(x, z)}{K(x, y)} d \omega_{x_{0}}(z) \\
& \leq M \int_{L_{2}} P_{z}^{y}\left(\text { ever hit } L_{j}\right) K(z, y) \frac{K_{2}(r, z)}{K(r, y)} d \omega_{x_{0}}(z) \\
& \leq M \int_{L_{2}} P_{z}^{y}\left(\text { ever hit } L_{j}\right) \frac{K(z, y)}{K(r, y)} d \omega_{r}(z) \\
& =M P_{r}^{y}\left(\text { ever hit } L_{j}\right) .
\end{aligned}
$$

Furthermore, we note that (2.6) for $x \in \partial D_{1} \backslash \partial D_{f}$ implies (2.6) for $x \in D_{1}$, by a simple conditioning argument. Thus, to prove (i), it suffices to show that (i) holds for $x=r$. We claim

$$
\frac{K(z, y)}{K(r, y)}<M, \quad z \in \partial D_{1} \backslash \partial D_{f} .
$$


This follows from Lemma 2.0 applied to $u(z)=\frac{K(z, y)}{K(r, y)}\left(\right.$ note $K(r, y) \leq M K\left(x_{0}, y\right)$ by the Harnack inequality), and the function $v(z)=P_{z}\left(B_{\tau_{D_{f}}} \in \partial D_{f} \backslash \partial D_{2}\right)$. It is easy to show $v(r)>\frac{1}{M}>0$. Now (2.7) implies

$$
\frac{K(z, y)}{K(r, y)}<M, \text { if } z \in D_{f} \backslash D_{1}
$$

and it is easily shown that $\omega_{r}^{j}\left(L_{j}\right) \leq \theta_{M}^{j-1}$, where $\theta_{M}<1$ and $\omega^{j}$ is the harmonic measure on $\partial D_{j}$ with respect to $D_{j}$, (see Davis and Zhang [5] e.g.) since this is easily shown if $D_{f}$ is a cone with vertex $y$. Thus

$$
\begin{aligned}
P_{r}^{y}\left(\text { ever hit } L_{j}\right) & \leq \int_{L_{j}} \frac{K(z, y)}{K(r, y)} d \omega_{r}^{j}(z) \\
& \leq M \theta_{M}^{j-1},
\end{aligned}
$$

which implies (i) for $x=r$.

Proof of (ii):

By scaling, it is equivalent to show that, for $x$ and $y$ outside $L_{0}$,

$$
P_{x}^{y}\left(\tau_{-m n_{0}}<\tau\right) \leq \frac{1}{2^{m}}
$$

We can use the same argument as that of the proof of (i) to show this.

The two-dimensional case of the following lemma is due to Burgess Davis [3], see also M. Cranston [2]. Here we only sketch an "analytic" proof for dimensions three and higher.

Lemma 2.2. Let $\Gamma$ be a domain of $\mathbb{R}^{d}, d \geq 2$, and $Q$ be a Whitney cube of $\Gamma$, then

$$
c|Q|^{2 / d} P_{Q} \leq E_{x}^{y} T_{Q} \leq C|Q|^{2 / d} P_{Q} .
$$

Proof. For $z \in Q$, Harnack's inequality applied to $K(\cdot, y)$ implies that

$$
\begin{aligned}
E_{z}^{y} T_{Q} & =\int_{Q} G_{\Gamma}(z, q) \frac{K_{\Gamma}(q, y)}{K_{\Gamma}(z, y)} d q \\
& \leq C \int_{Q} G_{\Gamma}(z, q) d q \\
& \leq C \int_{B(z, \sqrt{d} \ell(Q))} \frac{1}{|z-q|^{d-2}} d q \\
& \leq C|Q|^{2 / d},
\end{aligned}
$$

where $B(z, r)$ is the ball of radius $r$ centered at $z$. Thus, using the strong Markov property at the time $Q$ is hit, we get

$$
E_{x}^{y} T_{Q} \leq C|Q|^{2 / d} P_{Q}
$$


If we let $\lambda Q$ be the scaling of $Q$ by $\lambda$ with respect to the center of $Q$, and let $P^{2 Q}$ and $E^{2 Q}$ stand for probability and expectation of Brownian motion killed at $\partial(2 Q)$, since $G_{2 Q} \leq G_{\Gamma}$, we obtain, for $z \in \partial Q$,

$$
\begin{aligned}
E_{z}^{y} T_{Q} & \geq c \int_{Q} G_{2 Q}(z, q) d q \\
& =c E_{z}^{2 Q} T_{Q} .
\end{aligned}
$$

Since $P_{z}^{2 Q}\left(\tau_{\left(\frac{1}{2} Q\right)^{c}}<\tau_{(2 Q)^{c}}\right)>c>0$, if $z \in \partial Q$,

$$
\begin{aligned}
E_{z}^{2 Q} T_{Q} & \geq c \inf _{w \in \partial\left(\frac{1}{2} Q\right)}\left\{E_{w}^{2 Q} T_{Q}\right\} \\
& \geq c \inf _{w \in \partial\left(\frac{1}{2} Q\right)}\left\{E_{w}^{2 Q} \tau_{B\left(w, \frac{1}{4} \ell(Q)\right)}\right\} \\
& \geq c|Q|^{2 / d} .
\end{aligned}
$$

Again, the strong Markov property at the time $Q$ is hit implies that

$$
E_{x}^{y} T_{Q} \geq c|Q|^{2 / d} P_{Q}
$$

Let $Q$ and $R$ be two Whitney cubes of $D$ and let $\lambda>0$. By scaling, if we use $\lambda Q_{i}, i \geq 0$, to partition $\lambda D$, where $Q_{i}, i \geq 0$, partition $D$ and $\lambda D$ is the usual scaling $D$ by $\lambda$, we have $\rho_{\lambda D}(\lambda Q, \lambda R)=\rho_{D}(Q, R)$ and $\operatorname{cov}_{\lambda x}^{\lambda y}\left(T_{\lambda Q}, T_{\lambda R}\right)=$ $\lambda^{4} \operatorname{cov}_{x}^{y}\left(T_{Q}, T_{R}\right)$. Thus we may and do assume from now on by scaling again, without loss of generality, that $\ell(R) \geq \ell(Q)=\frac{1}{M}$, where $\frac{1}{M}$ is a small positive constant depending only on $M$, and that $d(Q, \partial D)=c_{M} d\left(Q, q_{0}\right)$, where $c_{M}>0$ and depends only on $M$ and $q_{0} \in \partial D$, just below the center of $Q$. Again, we may assume that $q_{0}=0$. Otherwise, we will let our "rectangles" $L_{a}$ be centered at $q_{0}$.

Lemma 2.3. There exists a positive constant $C_{M}$ such that if $\rho(Q, R) \geq C_{M} k$, for a positive integer $k$, then

$$
d(Q, R) \geq 2^{10 k} .
$$

Proof. If $d(Q, R)<2^{10 k}$, then it is not hard to show that there exists a Whitney chain connecting $Q$ and $R$ of length less than $C_{M} k$, for some $C_{M}>0$, all cubes of which lie in two cones of $D$ containing $Q$ and $R$ respectively, with vertices at the boundary of $D$, and with apertures $\theta_{M}>0$. It is not hard to argue that the natural chain thus constructed has at most $C_{M} k$ cubes, so that $\rho(Q, R) \leq C_{M} k$.

We set $L_{a}^{\prime}=L_{n_{0} a}, D_{a}^{\prime}=D_{n_{0} a}$. Now by the assumptions on $Q$ that $\ell(Q)=\frac{1}{M}$ and $d(Q, \partial D)=c_{M} d(Q, 0)$, we know that $Q$ is inside $L_{3}^{\prime}$, and it follows immediately from this lemma that if $\rho(Q, R) \geq C_{M} k$, for a large positive integer $k$ and some constant $C_{M}$ being the product of the constant of Lemma 2.3 and $n_{0}$, then $R$ is outside $L_{9 k}^{\prime}$. For any $x \in D$ and $y \in \partial D$ fixed, it is easy to see that there is a number $s_{0}>0$ such that $Q$ is inside $L_{s_{0}}^{\prime}$ and $R$ is outside $L_{s_{0}+2 k}^{\prime}$, and furthermore neither $x$ nor $y$ belongs to that part of $D$ lying between $L_{s_{0}}^{\prime}$ and $L_{s_{0}+2 k}^{\prime}$. By scaling, we may assume that $s_{0}=0$. The following lemma comes from [9]. 
Lemma 2.4. Let $\delta>2, \mu$ be a positive finite measure on a set $S$, and $\theta$ be a measurable function on $S$ such that $0<a \leq \theta \leq A$. If we denote

$$
\begin{aligned}
B(\theta) & =\sup \left\{\int_{S} \theta(s) W(s) d \mu(s) / \int_{S} W(s) d \mu(s): \delta^{-1}<W<\delta\right\}, \\
b(\theta) & =\inf \left\{\int_{S} \theta(s) W(s) d \mu(s) / \int_{S} W(s) d \mu(s): \delta^{-1}<W<\delta\right\}, \\
\text { then } \frac{B(\theta)}{b(\theta)}-1 & \leq\left(1-\frac{1}{4} \delta^{-2}\right)\left(\frac{A}{a}-1\right) .
\end{aligned}
$$

Let $\Omega_{j}=D_{2 k-j}^{\prime}, S_{j}=L_{2 k-j}^{\prime}$, for $j=1,2, \ldots, k$. Let $K^{j}$ and $\omega_{j}$ be respectively the Martin kernel function and harmonic measure for $\Omega_{j}$ at the fixed reference point $x_{0}$ in $D_{0}$. Then we have the following lemma. As earlier, we sometimes use $M$ to stand for any constant depending only on the Lipschitz constant $M$ of $f$.

Lemma 2.5. For any $j \in\{1,2, \ldots, k\}$, any $x \in \Omega_{j+1}$, and any $z, \tilde{z} \in S_{j}$, we have

$$
M^{-1} \leq \frac{K^{j}(x, z)}{K^{j}(x, \tilde{z})} \leq M
$$

Proof. First for $z, \tilde{z} \in S_{j}$ such that $d(z, \tilde{z}) \leq d(x, z)$, by Theorem 5.20 of [9], we have

$$
M^{-1} \leq \frac{K^{j}(x, z)}{K^{j}(x, \tilde{z})} \leq M .
$$

Now there is an absolute constant $N$ such that there are $N$ points $z_{1}=z, z_{2}, \ldots$, $z_{N}=\tilde{z}$ of $S_{j}$ and for each pair $z_{i}, z_{i+1}$ we have $d\left(z_{i}, z_{i+1}\right) \leq d\left(z_{i}, x\right), i=1,2, \ldots$, $N-1$, therefore

$$
M^{-1} \leq \frac{K^{j}\left(x, z_{i}\right)}{K^{j}\left(x, z_{i+1}\right)} \leq M, \quad i=1,2, \ldots, N-1 .
$$

Thus

$$
M^{-N} \leq \frac{K^{j}(x, z)}{K^{j}(x, \tilde{z})}=\prod_{i=1}^{N-1} \frac{K^{j}\left(x, z_{i}\right)}{K^{j}\left(x, z_{i+1}\right)} \leq M^{N} .
$$

Denote $M^{N}$ by $M$ again, since it only depends on $M$. We are done.

Lemma 2.6. There exists a positive constant $\eta=\eta_{M}<1$ such that for any $x, q$ inside $L_{0}$ and $y \in \partial D, y, r$ outside $L_{2 k}^{\prime}$ if we let $H(x, z)=H_{y}(x, z)=$ $G(x, z) \frac{K(z, y)}{K(x, y)}, z \in D$, then we have

$$
\left|\frac{H(x, r)}{H(q, r)}-1\right| \leq C \cdot \eta^{k}
$$

Proof. For any $j \in\{1,2, \ldots, k\}$, let $\tilde{z} \in S_{j}$ be fixed and for any $z \in S_{j}$, we define

$$
\begin{aligned}
W(z) & =W^{j}(z)=\frac{K^{j}(x, z)}{K^{j}(x, \tilde{z})}, \\
\theta(z) & =\frac{H(z, r)}{H(q, r)}, \text { and } \\
d \mu(z) & =K(z, y) d \omega_{j}(z) .
\end{aligned}
$$


Now the boundary Harnack principle [9] implies that

$$
M^{-1}<\theta(z)<M, \text { for } z \in S_{j} .
$$

Lemma 2.5 tells us that $M^{-1}<W(z)<M$, for $z \in S_{j}$. Since $G, K$ are harmonic, we have

$$
\begin{aligned}
G(x, r) & =\int_{S_{j}} K^{j}(x, z) G(z, r) d \omega_{j}(z) \text { and } \\
K(x, y) & =\int_{S_{j}} K^{j}(x, z) K(z, y) d \omega_{j}(z) .
\end{aligned}
$$

Thus,

$$
\frac{H(x, r)}{H(q, r)}=\frac{\int_{S_{j}} W(z) \theta(z) d \mu(z)}{\int_{S_{j}} W(z) d \mu(z)} .
$$

If we let

$$
\begin{aligned}
b_{j} & =\inf \left\{\theta(z): z \in S_{j}\right\} \text { and } \\
B_{j} & =\sup \left\{\theta(z): z \in S_{j}\right\},
\end{aligned}
$$

then

$$
b_{j+1} \geq \inf \left\{\frac{\int_{S_{j}} \theta(z) F(z) d \mu(z)}{\int_{S_{j}} F(z) d \mu(z)}: M^{-1}<F<M\right\}
$$

and

$$
B_{j+1} \leq \sup \left\{\frac{\int_{S_{j}} \theta(z) F(z) d \mu(z)}{\int_{S_{j}} F(z) d \mu(z)}: M^{-1}<F<M\right\} .
$$

Lemma 2.4 implies that

$$
\frac{B_{j+1}}{b_{j+1}}-1 \leq\left(1-\frac{1}{4} M^{-2}\right)\left(\frac{B_{j}}{b_{j}}-1\right) .
$$

Thus,

$$
\left|\frac{B_{j}}{b_{j}}-1\right| \leq C^{2}\left(1-\frac{1}{4} M^{-2}\right)^{j} .
$$

Let $\eta_{M}=1-\frac{1}{4} M^{-2}$. We have, by the maximal principle,

$$
\left|\frac{H(z, r)}{H(q, r)}-1\right| \leq C \cdot \eta^{j}, \text { for } z \in \Omega_{j}, j=1, \ldots, k .
$$

Therefore

$$
\left|\frac{H(x, r)}{H(q, r)}-1\right| \leq C \cdot \eta^{k} .
$$




\section{Covariance estimates}

The following proposition essentially handles the case where $\rho(Q, R)$ is small.

Proposition 3.1. If $Q$ and $R$ are Whitney cubes of $D$ with disjoint interiors, then

$$
\left|\operatorname{cov}\left(T_{Q}, T_{R}\right)\right| \leq C|Q|^{2 / d}|R|^{2 / d}\left(P_{Q}+P_{R}\right) \text {. }
$$

Proof. If we let

$$
\begin{aligned}
I & =\int_{Q} \int_{R} G(q, r) G(x, r) \frac{K(q, y)}{K(x, y)} d q d r, \\
I I & =\int_{Q} \int_{R} G(q, r) G(x, q) \frac{K(r, y)}{K(x, y)} d q d r, \\
\text { and } \quad I I I & =\int_{Q} \int_{R} G(x, q) G(x, r) \frac{K(q, y) K(r, y)}{K(x, y)^{2}} d q d r,
\end{aligned}
$$

then (1.1) implies that

$$
\operatorname{cov}\left(T_{Q}, T_{R}\right)=I+I I-I I I .
$$

Together with the fact that $P_{R} \cdot \max _{r \in R} P_{r}^{y}(Q) \leq C P_{Q}$, Lemma 2.2 implies that

$$
\begin{aligned}
I & =\int_{R} G(x, r) \frac{K(r, y)}{K(x, y)}\left(\int_{Q} G(q, r) \frac{K(q, y)}{K(r, y)} d q\right) d r \\
& \leq C|R|^{2 / d} P_{R}\left(\max _{r \in R} P_{r}^{y}(Q)|Q|^{2 / d}\right) \\
& \leq C|Q|^{2 / d}|R|^{2 / d}\left(P_{Q}+P_{R}\right), \\
I I & =\int_{Q} G(x, q) \frac{K(q, y)}{K(x, y)}\left(\int_{R} G(q, r) \frac{K(r, y)}{K(q, y)} d r\right) d q \\
& \leq C|Q|^{2 / d} P_{Q}\left(\max _{q \in Q} P_{q}^{y}(R)|R|^{2 / d}\right) \\
& \leq C|Q|^{2 / d}|R|^{2 / d}\left(P_{Q}+P_{R}\right),
\end{aligned}
$$

and

$$
\begin{aligned}
I I I & =\int_{Q} G(x, q) \frac{K(q, y)}{K(x, y)} d q \int_{R} G(x, r) \frac{K(r, y)}{K(x, y)} d r \\
& \leq C|Q|^{2 / d}|R|^{2 / d} P_{Q} \cdot P_{R} \\
& \leq C|Q|^{2 / d}|R|^{2 / d}\left(P_{Q}+P_{R}\right) .
\end{aligned}
$$

Therefore, $\left|\operatorname{cov}\left(T_{Q}, T_{R}\right)\right| \leq C|Q|^{2 / d}|R|^{2 / d}\left(P_{Q}+P_{R}\right)$.

If $Q$ and $R$ have common interiors, then $Q=R$ for $Q, R \in W(D)$. The proof of Proposition 3.1 implies that $E_{x}^{y} T_{Q}^{p} \leq C_{p}\left(|Q|^{2 / d}\right)^{p} \cdot P_{Q}$, for $p>0$.

For $p=2$, we have $E_{x}^{y} T_{Q}^{2} \leq C\left(|Q|^{2 / d}\right)^{2} \cdot P_{Q}$, so

$$
\begin{aligned}
\left|\operatorname{cov}\left(T_{Q}, T_{Q}\right)\right| & \leq\left(E_{x}^{y} T_{Q}^{2}\right)+\left(E_{x}^{y} T_{Q}\right)^{2} \\
& \leq C|Q|^{2 / d} \cdot|Q|^{2 / d}\left(P_{Q}+P_{Q}\right) \\
& =C\left(|Q|^{2 / d}\right)^{2} \cdot P_{Q} .
\end{aligned}
$$

With the following proposition that essentially handles the case where $\rho(Q, R)$ is large, we can complete the proof of Theorem 1.2. 
Proposition 3.2. If $Q$ and $R$ are Whitney cubes of $D$ with disjoint interiors, satisfying $\rho(Q, R) \geq C_{M} \cdot m$, where $C_{M}$ is the constant in Lemma 2.3 times $n_{0}$ and $m$ is a large integer, then

$$
\left|\operatorname{cov}\left(T_{Q}, T_{R}\right)\right| \leq C_{M} e^{-C_{M} \rho(Q, R)}|Q|^{2 / d}|R|^{2 / d}\left(P_{Q}+P_{R}\right) .
$$

Proof. Using the same ideas that inspired the comments just after the proof of Lemma 2.3, we assume without loss of generality that $x$ and $Q$ are both inside $L_{0}$ and $R$ is outside $L_{3 m}^{\prime}$, and that either: (i) $y$ is outside $L_{3 m}^{\prime}$, or (ii) $y$ is inside $L_{0}$.

First we assume that $y$ is outside $L_{3 m}^{\prime}$. Harnack's inequality and Lemma 2.1 imply that $\max _{r \in R} P_{r}^{y}(Q) \leq c \eta^{m}$.

$$
\begin{aligned}
I & =\int_{R} G(x, r) \frac{K(r, y)}{K(x, y)}\left(\int_{Q} G(q, r) \frac{K(q, y)}{K(r, y)} d q\right) d r \\
& \leq \int_{R} G(x, r) \frac{K(r, y)}{K(x, y)}\left(C|Q|^{2 / d} P_{r}^{y}(Q)\right) d r \\
& \leq C|R|^{2 / d} P_{R} \cdot|Q|^{2 / d} \max _{r \in R} P_{r}^{y}(Q) \\
& \leq C \eta^{m}|Q|^{2 / d}|R|^{2 / d}\left(P_{Q}+P_{R}\right),
\end{aligned}
$$

and

$$
\begin{aligned}
|I I-I I I| & =\left|\int_{Q} \int_{R} G(x, q) \frac{K(q, y)}{K(x, y)} K(r, y)\left[\frac{G(r, q)}{K(q, y)}-\frac{G(x, r)}{K(x, y)}\right] d q d r\right| \\
& =\left|\int_{Q} \int_{R} G(x, q) \frac{K(q, y)}{K(x, y)} \cdot G(x, r) \frac{K(r, y)}{K(x, y)}\left[\frac{H(x, r)}{H(q, r)}-1\right] d q d r\right| \\
& \leq C \eta^{m} \int_{R} G(x, r) \frac{K(r, y)}{K(x, y)} d r \int_{Q} G(x, q) \frac{K(q, y)}{K(x, y)} d q \\
& \leq C \eta^{m} \cdot|Q|^{2 / d}|R|^{2 / d} P_{Q} \cdot P_{R} \\
& \leq C_{M} e^{-C_{M} \rho(Q, R)}|Q|^{2 / d}|R|^{2 / d}\left(P_{Q}+P_{R}\right) .
\end{aligned}
$$

The first inequality comes from Lemma 2.6 and the second from Lemma 2.2. Therefore, by (1.1),

$$
\left|\operatorname{cov}\left(T_{Q}, T_{R}\right)\right| \leq C_{M} e^{-C_{M} \rho(Q, R)}|Q|^{2 / d}|R|^{2 / d}\left(P_{Q}+P_{R}\right) .
$$

Next we assume $y$ is inside $L_{0}$. This case is relatively easy to prove. The strong Markov property implies that

$$
\begin{aligned}
E_{x}^{y} T_{Q} T_{R} \leq & E_{x}^{y}\left(E_{Z_{\tau_{Q}}}^{y} T_{Q} T_{R} I\left(\tau_{Q^{c}}<\tau\right)\right) \\
& +E_{x}^{y}\left[T_{R}\left(E_{Z_{\tau_{Q^{c}}}}^{y} T_{Q}\right) I\left(\tau_{Q^{c}}<\tau\right)\right] \\
\leq & C|Q|^{2 / d}|R|^{2 / d} \cdot \max _{q \in Q} P_{q}^{y}(R) \cdot P_{Q} \\
& +C|Q|^{2 / d}|R|^{2 / d} \cdot \sqrt{P_{R}} \cdot P_{Q} \\
\leq & C(\sqrt{\eta})^{m}|Q|^{2 / d}|R|^{2 / d}\left(P_{Q}+P_{R}\right) \\
\leq & C_{M} e^{-C_{M} \rho(Q, R)}|Q|^{2 / d}|R|^{2 / d}\left(P_{Q}+P_{R}\right),
\end{aligned}
$$


the second inequality comes from the Schwartz inequality and (3.1). Therefore,

$$
\begin{aligned}
\left|\operatorname{cov}\left(T_{Q}, T_{R}\right)\right| & \leq E_{x}^{y} T_{Q} E_{x}^{y} T_{R}+E_{x}^{y} T_{Q} T_{R} \\
& \leq C_{M} e^{-C_{M} \rho(Q, R)}|Q|^{2 / d}|R|^{2 / d}\left(P_{Q}+P_{R}\right) .
\end{aligned}
$$

This completes the proof of Theorem 2.1. Finally, we sketch an analytic proof of Theorem 1.1. Note that covariance formula (1.1) is true for any Greenian domain of any dimension. If $\phi$ is a conformal mapping from a simply connected planar domain $D$ to the strip

$$
S=\left(-\frac{\pi}{2}<\operatorname{Im}(z)<\frac{\pi}{2}\right)
$$

and $\psi(z)=e^{z+i \frac{\pi}{2}}$ conformally maps the strip to the upper-half plane $R_{+}^{2}$, we let $\Phi=\psi(\phi), \Psi=\Phi^{-1}$, and $w^{\prime}=\Phi(w)$. If $\rho(Q, R) \geq c m$, where $c$ is an absolute constant that is bigger than $c_{2}$, the constant in Davis' Lemma 4.2 of [3], and $m$ is an integer, then, together with this lemma, scaling implies that $\Phi(Q)$ is in $L_{0}$ and $\Phi(R)$ is outside $L_{3 m}$ and that furthermore neither $\Phi(x)$ nor $\Phi(y)$ belongs to that part of $R_{+}^{2}$ lying between $L_{0}$ and $L_{3 m}$. We may assume that $\Phi(x)$ is inside $L_{0}$. Similarly, we have two cases to consider: (i) $\Phi(y)$ is inside $L_{0}$; (ii) $\Phi(y)$ is outside $L_{3 m}$.

First we assume that $y^{\prime}$ is outside $L_{3 m}$. The proof of (i) of Lemma 2.1 implies that $\max _{r \in R} P_{r}^{y}(Q) \leq c \eta^{m}$. By Lemma 2.2,

$$
\begin{aligned}
I & =\int_{R} G(x, r) \frac{K(r, y)}{K(x, y)}\left(\int_{Q} G(q, r) \frac{K(q, y)}{K(r, y)} d q\right) d r \\
& \leq \int_{R} G(x, r) \frac{K(r, y)}{K(x, y)}\left(C|Q| P_{r}^{y}(Q)\right) d r \\
& \leq C|R| P_{R} \cdot|Q| \max _{r \in R} P_{r}^{y}(Q) \\
& \leq C \eta^{m}|Q||R|\left(P_{Q}+P_{R}\right),
\end{aligned}
$$

and

$$
\begin{aligned}
& |I I-I I I|=\left|\int_{Q} \int_{R} G(x, q) \frac{K(q, y)}{K(x, y)} K(r, y)\left[\frac{G(r, q)}{K(q, y)}-\frac{G(x, r)}{K(x, y)}\right] d q d r\right| \\
& =\mid \int_{Q^{\prime}} \int_{R^{\prime}} G\left(x^{\prime}, q^{\prime}\right) \frac{K\left(q^{\prime}, y^{\prime}\right)}{K\left(x^{\prime}, y^{\prime}\right)} \cdot G\left(x^{\prime}, r^{\prime}\right) \frac{K\left(r^{\prime}, y^{\prime}\right)}{K\left(x^{\prime}, y^{\prime}\right)}\left[\frac{H\left(x^{\prime}, r^{\prime}\right)}{H\left(q^{\prime}, r^{\prime}\right)}-1\right] \\
& \quad \cdot\left|\Psi^{\prime}\left(q^{\prime}\right)\right|^{2}\left|\Psi^{\prime}\left(r^{\prime}\right)\right|^{2} d q^{\prime} d r^{\prime} \mid \\
& \leq C \eta^{m} \int_{R^{\prime}} G\left(x^{\prime}, r^{\prime}\right) \frac{K\left(r^{\prime}, y^{\prime}\right)}{K\left(x^{\prime}, y^{\prime}\right)}\left|\Psi^{\prime}\left(r^{\prime}\right)\right|^{2} d r^{\prime} \int_{Q^{\prime}} G\left(x^{\prime}, q^{\prime}\right) \frac{K\left(q^{\prime}, y^{\prime}\right)}{K\left(x^{\prime}, y^{\prime}\right)}\left|\Psi^{\prime}\left(q^{\prime}\right)\right|^{2} d q^{\prime} \\
& \leq C \eta^{m} \int_{R} G(x, r) \frac{K(r, y)}{K(x, y)} d r \int_{Q} G(x, q) \frac{K(q, y)}{K(x, y)} d q \\
& \leq C \eta^{m} \cdot|Q \| R| P_{Q} \cdot P_{R} \\
& \leq C e^{-c \rho(Q, R)}|Q \| R|\left(P_{Q}+P_{R}\right) .
\end{aligned}
$$


The first inequality comes from Lemma 2.6 and the third from Lemma 2.2. Therefore, by (1.1),

$$
\left|\operatorname{cov}\left(T_{Q}, T_{R}\right)\right| \leq C e^{-c \rho(Q, R)}|Q \| R|\left(P_{Q}+P_{R}\right) .
$$

Next we assume $y^{\prime}$ is inside $L_{0}$. The proof of this case is the same as that of Theorem 1.2 and the only difference is that in the two-dimensional case the constants are absolute.

\section{ACKNOWLEDGEMENTS}

This paper is part of the author's doctoral dissertation, written under the guidance of Burgess Davis. I would like to thank him for his help.

\section{REFERENCES}

[1] R. Bañuelos and B. Davis, A Geometrical Characterization of Intrinsic Ultracontractivity for Planar Domains with Boundaries Given by Graphs of Functions, Indiana University Mathematical Journal 41 (1992), 885-913. MR 94g:60142

[2] M. Cranston, Conditional Brownian Motion, Whitney Squares and the Conditional Gauge Theorem, Seminar on Stochastic Processes, Birkhäuser 17 (1988), 109-119. MR 90j:60078

[3] B. Davis, Conditioned Brownian Motion in Planar Domains, Duke Math. J. 59 (1988), 397-421. MR 89j:60112

[4] B. Davis, Intrinsic Ultracontractivity for Dirichlet Laplacian, J. Funct. Anal. 100 (1991), 163-180. MR 92k:35065

[5] B. Davis and B. Zhang, Moments of the Lifetime of Conditioned Brownian Motion in Cones, Proceedings of the American Mathematical Society 121 (1994), 925-929. MR 94i:60097

[6] J.L. Doob, Classical Potential Theory and its Probabilistic Counterpart, Springer-Verlag, Berlin, 1984. MR 85k:31001

[7] R.A. Hunt and R.L. Wheeden, Positive Harmonic Functions on Lipschitz Domains, Trans. Amer. Math. Soc. 132 (1968), 307-322. MR 43:547

[8] P.W. Jones, Extension Theorems for BMO, Indiana Univ. Math. J. 29 (1980), 41-66. MR 89b: 42047

[9] D.S. Jerison and C.E. Kenig, Boundary Value Problems on Lipschitz Domain, M.A.A. Studies in Math., Studies in Partial Differential Equations, Walter Littman 23 (1982), 1-68. MR 85f: 35057

[10] E.M. Stein, Singular Integrals and Differentiability Properties of Functions, Princeton Univ. Press, Princeton, N.J., 1970. MR 44:7280

Department of Mathematics, Purdue University, West Lafayette, Indiana 47907

E-mail address: biao@math.purdue.edu 